\title{
MALE URETHRAL STRICTURES : MANAGEMENT OPTIONS
}

\author{
ZAHID HASSAN BHUIYAN
}

\begin{abstract}
: $1,2,3,4,5$
Controlled clinical trials are unusual in surgery, rare in urology, and almost non existent as far as the management of urethral stricture is concerned. What data there are come largely from so called "expert opinion" and the quality of this is variable. None the less, the number of so called experts, past and present, is comparatively small and in broad principle their views more or less coincide. Although this review is therefore inevitably biased, it is unlikely that expert opinion will take issue with most of the general points raised here.
\end{abstract}

Keywords: urethral stricture

Bangladesh J. Urol. 2018; 21(2): 158-163

Urethral stricture is well known since ancient civilization. Indeed not much has changed of its management until about 50 years ago.

Statistics in USA suggest that men are affected with an increasing incidence from about 1 in every 10000 men aged 25 to about 1 in every 1000 men ages 65 or more. In the past stricture were caused by gonococcal urethritis. Now a days the causes are inflammatory, traumatic, idiopathic, and also congenital. The inflammation category includes non specific urethritis and increasingly commonly lichen sclerosus (LS), for merely known as balanitis xerotica obliterans (BXO). Trauma is more common cause now a days, usually iatrogenic (urethral instrumentation, post hypospadias surgery), fall on astride lead to injuries to the bulbar urethra, pelvic fracture related injuries to the membranous urethra and bulbo membranous junction. Many strictures are "idiopathic" because no specific causes could be attributable for this. Many of these occur at the junction of the proximal and middle sections of the bulbar urethra in adolescents and young adults. However congenital strictures are well documented among children.

The pathology of urethral stricture disease is poorly understood in most cases. External trauma generally

1. Professor, Department of Urology, Bangladesh Medical College \& Hospital

Correspondences: Professor Dr. Md. Zahid Hassan Bhuiyan, Department of Urology, Bangladesh Medical College \& Hospital, Email : zahidhb_1961@yahoo.com, Cell : 8801534622364

Received: 9 August 2017

Accepted: 05 March 2018 causes partial or complete disruption of an otherwise normal urethra-that is clear. But it would seem that for whatever reason a scar develops as a consequence of changes in the structure and function of the urethral epithelium and the sub epithelial spongy tissue causing a fibrotic narrowing of the urethra. Secondary changes in the epithelium more proximally develop there afterwards causing a progressive stricturing of an increasing length of the urethra from before backwards. Longstanding urethral obstruction may cause secondary complications in the rest of the urinary tract.

\section{Clinical Presentation: ${ }^{1}$}

Most patients present with slow and progressive deterioration of the urinary stream leading to a feeling of incomplete emptying of bladder. There is also splaying of the urinary stream, increase frequency, urgency, straining and urethralgia during micturation. In PTSU most patients can't void and carry a suprapubic catheter.

After reviewing history both present and past, physical examination may reveal LS, meatal stenosis, local redness, urethral discharge, one or more areas are hard or swollen, abscess, fistuae in the corpus spongiosum.

Patients may present with features of complication; into acute or chronic urinary retention, prostatitis, bladder dysfunction, urethral diverticulum, periurethral abscess, epidedymo-orchitis, fournier's gangrene, urethral 
fistula, bilateral hydronephrosis, urinary infections, urinary calculus formation etc.

\section{Investigations ${ }^{1,6,7}$}

The simple office procedure is Uroflometry. Typically the peak flow rate is low but the flow pattern is characteristically flat. The flexible cystoscopy is another office procedure.

Retrograde urethrogram + micturating cystourethrogram (RGU+MCU) in the next step of investigation. If properly performed, this will show the exact site and length of the stricture. In case of complete stricture, the additional procedure is antigrade cystogram, through suprapubic catheter tract. In some case, the internal urethral sphincter and posterior urethra is not well visualized; in those cases antigrade flexible cysto-urethroscopy through suprapubic catheter tract can nicely assess the bladder neck and prostatic urethra proximally.

Ultrasonography of the male urethra can be useful in evaluating urethral strictures. Interms of stricture length, the degree and depth of spongiofibrosis. Ouattara et al (2004) showed that urethral strictures identified on perineal sonograms were significantly longer than those identified on RGU + MCU. In addition changes in the bladder wall, presence of residual urine, hydroureteronephrosis due to longstanding outflow obstruction can also be assessed.

MRI is considered to be the best ancillary imaging modality for the assessment of stricture, associated with pelvic fracture (PTSU). It can delineate the details of stricture as well as pelvic anatomy.

\section{Treatment modalities}

Dilatation, urethrotomies (DVIU, LASER) approaches. $7,8,9,10$

The principle of urethral dilatation is to stretch the urethral stricture up. A graduated series of dilators from smaller to larger size is passed to restore the urethral caliber to normal. As a general rule urethral dilatation might best be reserved for more straightforward strictures that can be dilated in an office setting using topical anaesthesia

Urethotomy : It is performed blindly by increasingly sophisticated means until direct visual internal urethrotomy (DVIU) was developed about 50 years ago. DVIU is probably better for more complicated but short strictures, performed in a day case in operating theatre, under regional or general anaesthesia. Alternatively the use of a laser rather than a cold knife for internal urethrotomy. Visual control of the process might be expected to give better results but in fact, there is no evidence that this is so. Whereas Both procedures are generally regarded as simple and straightforward but in fact carry a significant risk of complications.

It is now generally accepted that urethrotomy and dilatation are equally effective and can be expected to cure about $50 \%$ of short bulbar urethral strictures when first used. If the procedure has to be repeated, it is rarely curative even the first time in strictures other than in the bulbar urethra.

However the use of clean intermittent self catheterization (CISC) after dilatation, DVIU, laser urethrotomy is helpful. It may prolong the recurrence. When the stricture recurs, it usually does so within weeks or months and almost always within two years. Those that recur comparatively infrequently might be palliated (rather than cured) by repeat instrumentation and, as long as this is agreeable to the patient and is uncomplicated by bleeding or sepsis, Such palliation is acceptable. If instrumentation is required more frequently or is complicated or unacceptable to the patient in the long run, then urethroplasty is the only curative option.

Urethral stents : Although popular in the past but long term outcome commonly do more harm than good. So was withdrawn from market.

In summary 12, 13, 14 urethral dilation and other endoscopic approaches such as direct vision internal urethrotomy (DVIU), laser urethrotomy, are usually for short urethral stricture. The recurrence are common. However the recurrence can be prevented or delayed (Cochrane review) by self intermittent urethral dilation. In some cases the stricture may worsen, making future attempts to surgically repair the urethra more difficult. Principles of urethroplasty 15,16

In case of short urethral strictures specially bulber or more proximal stricture (limitations of elasticity and length), excision and end to end anastomosis, or an anastomotic urethroplasty, gives the best and most sustained results in terms of both a low re stricture rate and a low complication rate.

When a stricture is too long for anastomotic urethroplasty, the alternative is some form of substitution urethroplasty. Here, there are two alternative approaches. The first is to excise the urethral stricture and perform a circumferential repair of the 
urethra. Intuitively, this would seem to be a sensible approach but in practice, a circumferential repair needs to be staged to give satisfactory results or, if performed in one stage, tends to give less satisfactory results.

The common practice for the same is incising the stricture and then putting on a patch to restore urethral calibre at that point (substitution urethroplasty I stricturotomy and patch).

\section{Anastomotic urethroplasty ${ }^{17,18}$}

Anastomotic urethroplasty is generally applicable in two situations. Firstly, for short strictures of the bulbar urethra, not more than 1-2 cm long, which are generally attributable to external trauma or are otherwise idiopathic in origin (possibly congenital). The external trauma is usually a fall in astride injury. The second situation is a pelvic fracture related injury of the membranous urethra or bulbo membranous junction.

The general principles here are after $3-6$ month of initial injury, when active inflammation of the injured area has been resolved, stabilization of pelvis (in case unstable pelvis) is ensured, only then anastomotic urethroplasty can be attempted. In such case, after excision of all fibrous tissue, the healthy ends of the urethra need to be tension free anastomosis. For the same bulbar urethra is to be mobilized fully, to overcome the gap caused by complete disruption because of external trauma. If the urethral mobilisation alone be insufficient to bridge the defect then the second general principle is to straighten out the natural curve of the bulbar urethra by a sequential series of manoeuvres including separation of the crura at the base of the penis, wedge pubectomy of the inferior pubic arch, and, if necessary, re routing of the urethra around the shaft of the shaft of the penis until the course of the bulbar urethra from the penoscrotal junction to the apex of the prostate is a straight line rather than the semi circle that it normally is.

\section{Nontransectenging bulber Urethroplasty ${ }^{19,20,21}$}

The anatomy of the urethra, particularly the bulbomembranous urethra, provides the basis for the non-transecting technique The dorsal aspect of the bulbomembranous urethra is devoid of corpus spongiosum, it has a concave configuration as it curves around the point of fusion of the corporal bodies; The advantage of dorsal stricturotomy is that incision is in relatively avascular plane preserving the spongiosal blood flow ventrally, furthermore there is sufficient length to allow closure of a longitudinal stricturotomy in horizonal plane without tension.
Once the stricturotomy has been made for short length stricture and if it is membraneous stricture, in such case the membrane is excised. The mucosal margins on either side of this are sutured and the longitudinal stricturotomy closed transversely. This is effectively a 'Heinke-Mikulicz'-type stricturoplasty;

If the stricture is longer $(>2 \mathrm{~cm})$, the scarred epithelium with the surrounding spongiofibrosis is carefully excised leaving the healthy underlying spongiosum, the stricture incised longitudinally. After all possible measures a tension free horizontaal repair is desirable. If not possible a dorsal patch substitution is the best alternative.

\section{Substitution Urethroplasty ${ }^{21}$}

Unlike anastomotic urethroplasty this can be used anywhere in the urethra specially for any strictures those are too long for an anastomnotic repair.

Stricturotomy and patch is generally procedure of choice unless a urethral segment positively needs to be excised, when the urethra is irretrievably scarred (LS), when it is irretrievably fibrotic, or in such rare situations such as with urethral arteriovenous malformations or tumours.

\section{Substitution Urethroplsty : Flap versus graft ${ }^{22,23,24}$}

A vascularised skin flap, would give better results than a free graft. Indeed, for most strictures the results of free graft is as good as with a flap. For longer strictures flap may be inadequate but longer free graft can be harvested which is a thin, full thickness with a dense sub dermal plexus such as full thickness graft of penile shaft skin or foreskin, a post auricular (from behind the ear) full thickness graft, or a buccal mucosal graft (from inside of the cheek).

Buccal mucosa is currently the preferred graft material, because structurally it is close to the urethral mucosa and it has least donor site morbidity.

Flap is a good choice when the local conditions are not favourable for a graft as in extensive scarring from previous surgery or active infection or previous radiotherapy or when the stricture is unusually long or trans sphincteric. In such cases a flap of local genital skin pedicled on the dartos layer of the penis (which is highly vascular) is preferred.

It is possible to do this in one stage, for short strictures in the most distal part of the penile urethra, but in other circumstances a two stage repair is safer and gives the best long term results. In such circumstances a 
graft is interposed as a flat plate between the two ends of the urethra where a segment has been excised. The flat plate is then tubularised to form a tube and closed in layers at a second stage three to six months later.

Management of panurethral stricture

\section{Staged urethroplasty 25,26}

Historically, two-stage urethroplasty was favored for the management of panurethral strictures. The Johanson's two-stage technique, first described in 1953. . Panurethral strictures were mainly treated by 2 stage procedures until 1990s. The primary concern with the two-stage Johanson's technique is that it relies on genital skin for the neourethra. Two stage surgery is not scientific in LS as this is a disease of genital skin. Any two-stage procedure with genital skin will have a high risk of recurrence secondary to incorporation of diseased skin in urethra. The Johanson staged repair has long fallen out of favor as first-line therapy. It can still be employed to salvage the most complex urethral strictures.

Previous failed urethral stricture repair complicates management due to fibrosis, impaired vascularity and limited urethra available for mobilization. Recurrent strictures $>1.5 \mathrm{~cm}$. in penile ur may difficult in single stage even with generous use of inferior pubectomy or the transpubic approach with certain modifications. Is suchethra a 2-stage procedure is a safer option. Overall, staged reoperation can offer a successful outcome for the majority of these complex strictures.

\section{Single stage urethroplasty (Kulkarni et al) ${ }^{27,28}$}

Pan-urethral stricture, a common urological problem on the South Asian population. It represents a particularly difficult challenge to manage. In India, Lichen Sclerosis (LS) is the most common cause of panurethral stricture, followed by iatrogenic causes. Panurethral strictures secondary to LS almost always involve the meatus. In iatrogenic strictures, the meatus is often spared.

Dorsal onlay buccal graft urethroplasty was popularized by Barbagli in 1996. For pan urethral stricture penile urethra was approached through circumcision incision and bulbar urethra through perineal incision. The penoscrotal junction would be a watershed area with technical difficulty.

Kulkarni et al, applied the penile invagination technique and performed dorsal onlay buccal graft urethroplasty for panurethral incision through perineum. By invagination, the full anterior urethra was visualized as a single unit. The concept of meatotomy with graft insertion from meatus till proximal bulb was now possible through a single incision in perineum.

A very recent multi-center study from high volume urethroplasty units (8 international centers) concluded that single stage buccal graft urethroplasty was more successful than 2 stage procedures. In case of panurethral stricture cambind flaps and graft have success rate but higher complication rates with flap alone.

Stricture urethra in paediatric population 29, 30, 31, $32,33,34,35$

Paediatric urethral stricture disease represents an uncommon but difficult urological problem. It is mostly acquired as congenital but infectious strictures are rare. In general management include urethral dilatation, endoscopic visual internal urethrotomy and open urethral reconstruction. Dilatation is often used as an initial treatment, with some success. Endoscopic urethrotomy in children has been described. Though high success rate $(86 \%)$ was clamed in some selected series but in other series poor long-term results have been reported.

Open reconstruction of urethral strictures in children follows the same principles as that in adults. However, children differ in having a more abdominally located bladder and prostate, and less capacious pelvis. Because of these differences anatomy, it has been claimed that transperineal urethroplasty, successful in adults, is technically more difficult in the confined perineum of a child. Rather trans perineal approach is preferred in complex cases.

In the 1970s Turner-Warwick popularized a delayed one-stage perineal approach, comprising urethral mobilization followed by bulboprostatic anastomosis, to bridge defects of up to $2.5 \mathrm{~cm}$. while substitution urethroplasty or transpubic urethroplasty continued to be used for longer defects or complex posterior urethral defects, respectively. Success rate using the perineal $(93 \%)$ and transpubic $(91 \%)$ approach for bulboprostatic anastomosis is satisfectory. Most failures were short strictures, at the anastomotic site, and responsive to optical urethrotomy or repeat perineal anastomotic repair. Buccal mucosa has proved to be a successful grafting material, especially as a patch, for long-segment strictures not amenable to anastomotic urethroplasty.

In conclusion, the reference standard for treating posttraumatic urethral strictures in children is transperineal 
one-stage bulboprostatic anastomosis. Development of the intercrural space and inferior pubectomy is important in achieving a tension-free anastomosis in patients with long strictures. This procedure should be attempted first in every case and a transpubic procedure used only if a tension-free anastomosis cannot be made through the perineum.

Summary : General principles of urethral stricture surgery ${ }^{1}$

- Urethral dilatation and DVIU and laser internal urethrotomy are equally effective.

- $50 \%$ of short bulbar strictures are cured by the very first dilatation or urethrotomy.

- Penile urethral strictures are rarely cured by dilatation or urethrotomy. Clean intermitant self catheterization ( $\mathrm{CISC}$ ) may delay the recurrence.

- If a patient develops a recurrent stricture after a previous urethrotomy or dilatation, however long the interval, further instrumentation is never curative. The only curative alternative is urethroplasty.

- Whenever a urethroplasty is necessary an anastomotic urethroplasty is preferable because it has a lower re stricture rate, success is maintained long term and the complication rate is lower. Almost all cases of PTSU are approach by this technique.

- Non - transecting bulber urethroplasty is successful in short segment stricture and non-traumatic cases.

- Substitution urethroplasty is necessary for longer bulbar strictures and all strictures of the penile urethra.

- For most urethral strictures requiring substitution urethroplasty a stricturotomy and patch is preferable to excision and a tube graft flap.

- Grafts and flaps are equally good in most circumstances.

- In the bulbar urethra grafts are quicker and easier.

- A dorsal stricturotomy and patch has a higher success rate and a lower complication rate than ventral stricturotomy and patch.

- Buccal mucosal grafting has advantages for stricturotomy and patch in the bulbar urethra.

- In the penile urethra a flap is quicker and easier.

- For pan - urethral stricture, Kulkarni's single stage substitution urethroplasty, using buccal mucosa, through perineal approach is a time tested technique.

- When a circumferential repair is required or in case of complex urethral stricture a two stage reconstruction is safer and more reliable than a one stage technique.

- Stricture management in paediatric age group almost follow the principles of adult urethroplasty, however the anatomy of pelvis in relation to bladder and prostate and urethra need special attention.

Future prospect in urethral stricture management ${ }^{1}$

Many urologists' hopes have been raised by developments in tissue engineering to provide an alternative source of material for repair of these difficult strictures.

\section{References :}

1. Mundy AR. Management of urethral strictures. Postgrad Med J. 2006 Aug; 82(970): 489-493.

2. Fuhse J, Knüpper M. Urethral stricture after transurethral resection. International Urology and Nephrology. March 1984, Volume 16, Issue 1, pp 33-38 (Cite as).

3. Palminteri, E.; Brandes, S. B.; Djordjevic, M. (2012). "Urethral reconstruction in lichen sclerosus". Curr Opin Urol. 22 (6): 478-483.

4. Chambers R, Baitera B. The anatomy of urethral stricture. BJU 1977(49) 545-551.

5. Romero Perez P, Mira Linares A. Complications of the lower urinary tract secondary to urethral stenosis. Actas Urol Esp 1996 (20) 786-793.

6. Yosif D. MyPACS.net: Post traumatic urethral stricture. Radiology Teaching Files $>$ Case 36829605, Kasr Aini hospital, Egypt.

7. Kawashima A, Sandler CM, Wasserman NF etal. Imaging of urethral disease: a pictorial review. Radiographics. 2004;24 Suppl 1 : S195-216.

8. Devereux M, Burfield G. Prolonged follow up of urethral strictures treated by intermittent dilatation. BJU 197042231-239.

9. Steenkamp J, Heyns C, De Kock M. Internal urethrotomy versus dilatation as treatment for male urethral strictures: a prospective, randomised comparison. J Urol 199715798-101.

10. Database of Systematic Reviews. 12: CD010258. doi:10.1002/14651858. CD010258. pub2. PMID 25523166. 
11. Badlani G, Press S M, Defaclo A. et al Urolume endourethral prosthesis for the treatment of urethral strictures disease: long term results of the North American multicenter urolume trial. Urology 1995, (45) 846-856.

12. Santucci $R$ and Eisenberg L: Urethrotomy has a much lower success rate than previously reported. J Urol 2010; 183: 1859.

13. Hudak SJ, Atkinson TH, Morey AF. Repeat transurethral manipulation of bulbar urethral strictures is associated with increased stricture complexity and prolonged disease duration. J Urol. 2012 May;187(5):1691-5.

14. Jackson, MJ; Veeratterapillay, R; Harding, CK; Dorkin, TJ (19 December 2014). "Intermittent selfdilatation for urethral stricture disease in males". The Cochrane Database of Systematic Reviews. 12: CD010258. doi:10.1002/14651858. CD010258.pub2. PMID 25523166

15. Mundy A. Results and complications of urethroplasty and its future. BJU 199371322325.

16. Andrich D E, Mundy A R. Urethral strictures and their surgical treatment. BJU Int 200086571-580.

17. Turner Warwick R. Urethral stricture surgery. In: Mundy A, ed. Current operative surgery-urology. London: Bailliere Tindall, 1988, 160-218.

18. Koraitim M. The lessons of 145 post traumatic posterior urethral strictures treated in 17 years. $\mathrm{J}$ Urol1995, (15)363-66.

19. Mundy A. Reconstruction of posterior urethral distraction defects. Atlas Urol Clin North Am1997, (165)1492-1495.

20. Gingu C, Dick A, Patrascoiu S, et al. Nontransecting augmented roof anastomosis: The technique of choice for long bulbar urethral strictures with limited spongiofibrosis. Eur Urol Suppl 2014;13:e77.

21. Bugeja S, Daniela EA, Mundy AR. Translational Andrology and Urology. Non-transecting bulbar urethroplasty. Reconstructive Urology Unit, University College London Hospital, London NW1 2BU, UK. Submitted Nov 11, 2014. Accepted for publication Jan 02, 2015.

22. Greenwell T, Venn S, Mundy A. Changing practice in anterior urethroplasty. BJU Int 199883631-635.
23. 22. Andrich D, Mundy A. The Barbagli procedure gives the best results for patch urethroplasty of the bulbar urethra. BJU Int 2001(88)385-389.

24. Mundy $A$. The long term results of skin inlay urethroplasty. J Urol 1972107977-980.

25. Al-Ali M, Al-Hajaj R. Johanson's staged urethroplasty revisited in the salvage treatment of 68 complex urethralstricture patients: presentation of total urethroplasty. Eur Urol 2001;39:268.

26. Wadhwa SN, Chahalr, Hemal AK, Gupta NP, Dogra PN, Seth A. Management of obliterative post-traumatic posterior urethral stricture after failed initial urethroplasty. J Urol, June 1998 Volume 159, Issue 6, Pages 1898-1902.

27. Management of panurethral strictures Kulkarni $P$, Joshi P, Surana S, Kulkarni AH, Reconstructive Urology Center, India Received 13 December 2015; accepted 6 January 2016 Available online 28 February 2016.

28. Warner JN, Malkawil, DM, Joshi PM, Kulkarni SB,Lazzeri M, Barbagli G, Mori R, Angermeier KW, Storme O, Campos R, Velarde L, Gomez RG, Han JS, Gonzalez CM, Martinho D, Sandul A, Martins FE, Santucci RA. A multi-institutional evaluation of the management and outcomes of long-segment urethral strictures. Urology 2015;85 June (6)):1483-7.

29. Hafez TA, El-Assmy A, Sarhan O, et al. Perineal anastomotic urethroplasty for managing posttraumatic urethral strictures in children: the longterm outcome. BJUI Feb 2005, Volume 95(3)403406.

30. Madgar I, Hertz M, Goldwasser B, Ora HB, Mani $\mathrm{M}$, Jonas $\mathrm{P}$. Urethral strictures in boys. Urology 1987; 30: 46- 9.

31. Scherz HC, Kaplan GW. Etiology, diagnosis, and management of urethral strictures in children. Urol Clin North Am 1990; 17: 389-95.

32. Morra MN, Das S. Traumatic stricture of prostatic urethra in a ten-year-old. Urology 1991; 38: 552-3.

33. Rosen MA, Nash PA, Bruce JE, McAninch JW. The actuarial success rate of surgical treatment of urethral strictures. J Urol 1994; 151 (Suppl): 360, A529.

34. Turner-Warwick R. A personal view of the management of traumatic posterior urethral strictures. Urol Clin North Am 1977; 4: 111-24.

35. Andrich DE, Mundy AR. Substitution urethroplasty with buccal mucosal grafts. J Urol 2001; 165: 1131-3. 